\title{
Bioinspired Electro-Permeable Glycans at Carbon: Fouling Control for Sensing in Complex Matrices
}

James A. Behan ${ }^{*}$ Adam Mylest, Alessandro Iannaci, Éadaoin Whelan, Eoin M. Scanlan ${ }^{*}$ and Paula E. Colavita*

School of Chemistry, CRANN and AMBER Research Centres, Trinity College Dublin, College Green, Dublin 2, Ireland

* Corresponding authors: colavitp@tcd.ie (Paula E. Colavita), Tel: +35318963562 and scanlae@tcd.ie (Eoin M. Scanlan), Tel: +35318962514.

$\$$ Authors contributed equally to this work. 


\begin{abstract}
The effect of glycan adlayers on the electrochemical response of glassy carbon electrodes was studied using standard redox probes and complex aqueous matrices. Aryldiazonium cations of aryl-lactoside precursors were used to modify glassy carbon via spontaneous and electrochemically assisted covalent grafting. Contact angle and fluorescence binding using Peanut Agglutinin (PNA) as a diagnostic lectin indicate that electrografting results in adlayers with greater glycan surface density than those obtained via spontaneous reaction. X-ray photoelectron spectroscopy with a fluorinated analog confirmed that electrografting results in multilayers of cross-linked aryl-lactosides. Adsorption studies with Bovine Serum Albumin (BSA) show that aryl-lactoside adlayers minimize unspecific protein adsorption. However, no significant differences were detected between spontaneous and electrografted layers in their ability to resist protein fouling despite their differences in coverage. Voltammetry studies show that spontaneous grafting has minimal effects on the response of standard redox probes in solution, whereas electrografting results in additional charge transfer impedance arising from increased electrode passivation. Bare and lactoside-modified carbon electrodes were tested for the detection of caffeine before and after prolonged exposure to coffee solutions. Spontaneous grafting was found to result in optimal properties by imparting antifouling performance in these complex matrices while preserving fast interfacial charge transfer.
\end{abstract}




\section{Introduction}

Surface-bound glycans hold potential as versatile tools for tailoring interfacial reactions of biomolecules at carbon surfaces via regulation of either specific or non-specific interactions. Glycans are involved in a diverse array of biological functions [1,2] including control of structure, conformational stability and intrinsic and extrinsic recognition, thus affecting important biological processes from cell communication and adhesion, to biofilm formation and inflammatory responses. This versatility can be harnessed to develop novel biointerfaces that deliver multifunctional responses. The ability to control interfacial interactions is extremely important in applications of carbon surfaces in complex fluids, as is the case in particular for sensing and diagnostics. Complex matrices in these applications typically contain a variety of biomolecules spanning a range of relevant concentrations, thus presenting challenging conditions for the development of smart interfaces. This has prompted significant interest in the recent literature on surface modification reactions for glycan functionalization, as a strategy for encoding affinity, sensitivity and specificity at transducing surfaces [3-7].

The chemistry of aryldiazonium cations [8-12] offers a facile route for the immobilization of glycans from solution to create chemically well-defined interfaces with functional saccharide groups. Work from our group has shown that aryldiazonium cations prepared from saccharidebearing aminophenol precursors as in Figure 1 [13], can be used to spontaneously form adlayers of mono- and di-saccharides from solution in a single step. This reaction yields covalent immobilization of glycans on a range of materials including carbon [13-15], polymers [16, 17], metals and alloys [13, 18]; furthermore, it has been extended to more complex glycans that display specific binding interactions with organic substrates, such as cyclodextrins [19]. Interestingly, we have found that spontaneously formed adlayers of mono- and di-saccharides 
can significantly reduce protein and biomass retention at surfaces, both in the laboratory and in the field $[14,16,18]$. In the case of carbon materials, specifically, we have demonstrated that despite these saccharide layers being of sub-nm thickness and roughness, and thus topographically well-defined, they result in significant reductions of protein adsorption at nanoplasmonic carbon sensors [15].

$$
\mathrm{X}=\mathrm{F} \text { or } \mathrm{H}
$$

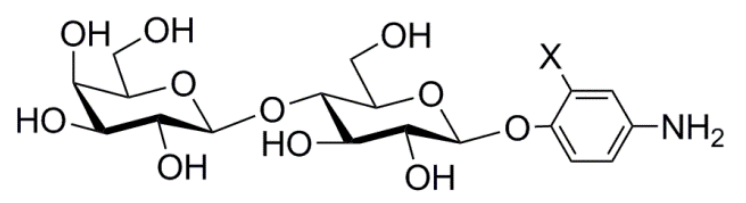

$$
\downarrow \begin{gathered}
\mathrm{NaNO}_{2} \\
\mathrm{HCl}
\end{gathered}
$$
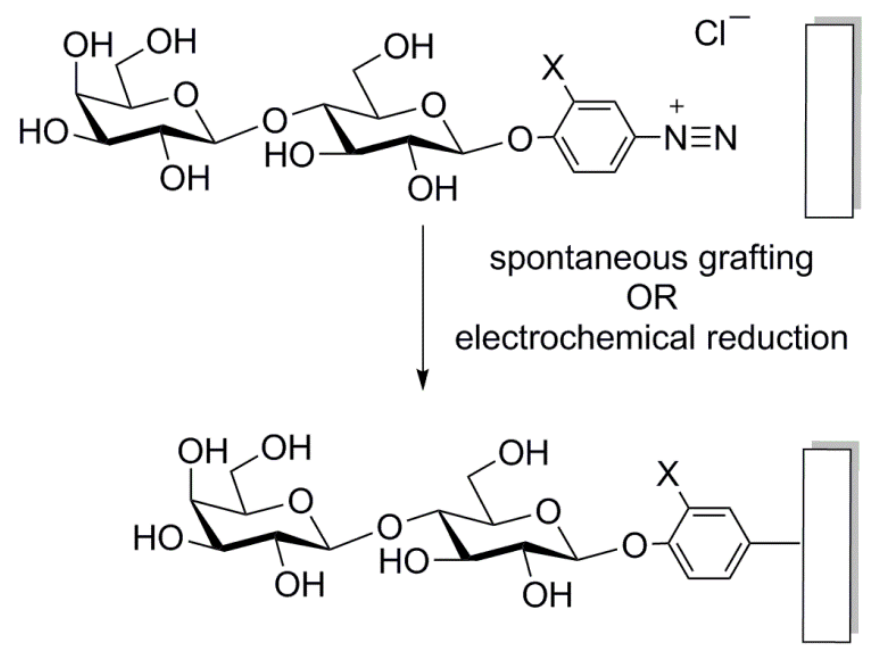

Figure 1. 4-aminophenol-O- $\beta$-D-galactopyranosyl $(1 \rightarrow 4)-\beta$-D-glucopyranoside precursor, its diazotization and subsequent covalent attachment to the carbon surface.

The above findings suggest that aryl-glycan functional layers might therefore offer a facile route to control unspecific adsorption and fouling in general, at carbon-based sensors and biosensors in aqueous media. Carbon materials are ubiquitous in electroanalysis due to their excellent bulk properties for electrochemical applications and the potential to tailor their surface 
to catalyse the redox response of a variety of analytes [20-23]. Fouling of the electroactive carbon surface is a common and challenging problem that can limit performance and lifetime of electrochemical sensors in applications that involve exposure to complex sample matrices. This has been highlighted in numerous reports of electroanalytical assays using carbon electrodes such as fibers, diamond, nanotubes and pyrolised resists [24-28]. However, to the best of our knowledge, saccharide modification has not been used to control unspecific adsorption at carbon electrodes.

In this work, we investigated the effect of glycan adlayers prepared via aryldiazonium chemistry on the electrochemical response of carbon using (a) standard redox probes in biomolecule-free solutions and (b) using a redox active analyte in a complex matrix. Functional adlayers can be used to prevent unspecific adsorption at carbon, however electroanalytical applications demand that fouling is minimised while preserving the electroactive interface necessary to sensing. Therefore functionalization protocols must be carefully tailored to ensure that fouling control is not achieved at the expense of surface passivation, which is detrimental to electrochemical performance.

To investigate the effect that glycan adlayer thickness and morphology have on the electrochemical response and fouling control at carbon, two electrode modifications were investigated: spontaneous grafting from solution, as discussed in previous work, and aryldiazonium electrografting. Electrochemically assisted grafting is a popular aryldiazonium functionalization strategy for conductive substrates [29-31], however it has no precedent in the immobilization of glycan derivatives of aryldiazonium cations. Electrochemical reduction of the aryldiazonium cation in solution results in $\mathrm{N}_{2}$ elimination and formation of a reactive aryl radical that can rapidly react with the electrode surface forming a covalent bond. Importantly, fast 
generation of aryl radicals via voltammetry or potential steps can result in grafting reactions to surface sites and to functional groups already bound to the surface and thus yields generally thick (10-20 nm), disordered multilayers via a 3-dimensional growth mechanism [32-35].

Our results show that spontaneously and electrografted aryl-lactoside layers differ significantly, with electrografting yielding multilayers despite lactoside groups being a relatively bulky substituent. The presence of the spontaneously formed lactoside layer does not significantly compromise the electrochemical response of the carbon electrode, whereas electrografting yields evidence of additional impedance to charge transfer at the carbon interface. Despite these significant differences, the two layers appear to minimize foulant retention to similar extents, as confirmed via tests using single protein and complex solutions such as beverage samples. These results are extremely promising as they indicate that aryldiazonium chemistry modification can be tailored to achieve electro-permeable adlayers to enable both fast charge transfer and fouling control in aqueous solutions.

\section{Experimental Methods}

\subsection{Chemicals and Materials}

Phosphate buffered saline buffer (0.010 M PBS, pH 7.4), sodium nitrite, hydrochloric acid, fluoroboric acid, glycerol $(>99.5 \%)$, formamide (>99.5\%), diiodomethane $(99 \%), \quad 1$ bromonaphthalene (97\%), peanut agglutinin from Arachis hypogaea (PNA) and bovine serum albumin (BSA) conjugates with fluorescein isothiocyanate (FITC) were purchased from SigmaAldrich; Sigradur K Glassy Carbon plates and Sigradur G Glassy Carbon discs were purchased from HTW. Hexaammine ruthenium(III) chloride (98\%), potassium chloride (Bioxtra, $>99.0 \%$ ) potassium hexacyanoferrate(II) (Analar, $>99 \%$ ) and Caffeine (Aldrich) were used without further 
purification. Aminophenol-lactoside compounds were synthesised as previously reported $[13,16$, 18]. Coffee for electroanalytical tests was prepared fresh (at $60{ }^{\circ} \mathrm{C}$ on output) before each experiment using tap water from a soft water region (Dublin 2, Ireland during spring 2018) using a Dolce Gusto ${ }^{\mathrm{TM}}$ Delongine coffee maker with a Nescafé Dolce Gusto ${ }^{\mathrm{TM}}$ Lungo instant coffee capsule.

\subsection{Surface Preparation}

Glassy carbon (GC) substrates were polished with $1,0.3$ and $0.05 \mu \mathrm{m}$ alumina slurries according to previously reported methods $[36,37]$. Spontaneous functionalization was carried out via immersion of GC substrates in freshly prepared $1.0 \mathrm{mM}$ solutions of aryldiazonium cations generated in situ from the 4-aminophenol-O- $\beta$-D-galactopyranosyl( $1 \rightarrow 4)$ - $\beta$-D-glucopyranoside precursor (Figure 1), following previously published protocols [13]. Briefly, a 1.25 $\mathrm{mM}$ solution of the 4-aminophenol in $0.00150 \mathrm{M} \mathrm{HCl}$ was prepared and cooled to $4{ }^{\circ} \mathrm{C}$ or less in an ice bath for $1 \mathrm{~h}$. The cold precursor solution was diluted via addition of $0.010 \mathrm{M} \mathrm{NaNO}_{2}$ to a final concentration of $0.0010 \mathrm{M}$ in the precursor. Samples were immersed into this solution and kept in the dark for $1 \mathrm{~h}$; they were then sonicated for $1 \mathrm{~min}$ each in $\mathrm{MeOH}$ and deionized water and were stored in Millipore water prior to further testing. Control GC surfaces for surface characterization were prepared by immersing the GC samples in solutions prepared as above, with the exception of addition of $\mathrm{NaNO}_{2}$.

Electrografting was carried out using the same precursor solution as for spontaneous grafting following a potentiostatic protocol described by Brooksby et al. [30]. The solution was deaerated with $\mathrm{Ar}$ and, subsequently, a potential step at $-0.5 \mathrm{~V}$ vs $\mathrm{Ag} / \mathrm{AgCl}$ ( $\mathrm{KCl}$ sat.) was applied for 300 $\mathrm{s}$; the duration of the potential step was chosen based on previous reports, that show this is typically sufficient to yield a limiting film on glassy carbon [30,38]. The reduction potential was 
chosen to be $c a .100 \mathrm{mV}$ cathodic to the peak in the cathodic wave obtained for the aryldiazonium reduction in cyclic voltammetry studies (Supporting Information). To minimize the effects of the spontaneous reaction on electrografted samples, the grafting was initiated immediately after immersion of the sample into the solution. The total charge passed during potentiostatic experiments was equivalent to the reduction of $(7 \pm 2) \times 10^{-8} \mathrm{~mol} \mathrm{~cm}^{-2}$ aryldiazonium cations (Supporting Information). Samples were sonicated for 1 min each in $\mathrm{MeOH}$ and water to remove loosely-bound material and stored in ultrapure water.

\subsection{Characterization}

Contact angles (CAs) were determined for all samples using the sessile drop method (FTA1000), using $2 \mu \mathrm{L}$ droplets. XPS characterization was performed at $1 \times 10^{-10}$ mbar base pressure in an Omicron ultrahigh-vacuum system using a monochromatized $\mathrm{Al} \mathrm{K} \alpha \mathrm{X}$-Ray source (1486.6 eV). Spectra were recorded at a $45^{\circ}$ takeoff angle with an analyzer resolution of $0.5 \mathrm{eV}$. Spectra were baseline-corrected using a Shirley background and fitted with Voigt functions using commercial software (CasaXPS); atomic percent compositions were determined by calculating peak area ratios after correction by relative sensitivity factors $(\mathrm{RSF}: \mathrm{C} 1 \mathrm{~s}=1.0, \mathrm{~N} 1 \mathrm{~s}=1.8, \mathrm{O} 1 \mathrm{~s}$ = 2.93). Electrochemical experiments were carried out using a Metrohm Autolab AUT50324 potentiostat using a 3-electrode setup as previously reported [36, 37, 39, 40]. A static disc holder (Pine Instruments) enclosing the GC disc electrode was used as working electrode while a saturated $\mathrm{Ag} / \mathrm{AgCl}$ ( $\mathrm{KCl}$ sat.) electrode and a graphite rod were used as reference and counter electrodes respectively. The electrodes were mounted in a glass cell fitted with a custom-made Teflon cap. All solutions were degassed with Ar gas prior to measurement and thermostated at $25^{\circ} \mathrm{C}$ in a water bath. 
Fluorescence imaging was carried out using glassy carbon plates as substrates, following published protocols [18]. The plates were immersed in 0.5 and $0.2 \mathrm{mg} \mathrm{mL}^{-1}$ solutions of the BSA-FITC and PNA-FITC conjugates, respectively, in PBS at $\mathrm{pH} 7.4 \mathrm{for} 1 \mathrm{~h}$; in the case of PNA-FITC the buffer contained also $0.1 \mathrm{mM} \mathrm{CaCl}_{2}$ and $\mathrm{MgCl}_{2}$. All samples were washed with PBS solution prior to imaging to remove excess unbound protein. Fluorescence images were acquired using an Olympus BX51 inverted microscope with CellSense digital image processing software. Emission intensities were analysed using $3^{\text {rd }}$ party software (ImageJ).

\section{Results and Discussion}

\subsection{Carbon Electrode Functionalization}

The properties of spontaneously grafted (GC-Lac-S) and electrografted (GC-Lac-E) aryldiazonium derived layers on GC were first characterized using a combination of water contact angle (WCA) and fluorescence microscopy measurements. Figure 2a shows a comparison of the WCA for unmodified GC and GC-Lac layers. A reduction in contact angle from $71 \pm 3^{\circ}$ to $46 \pm 2^{\circ}$ was observed following spontaneous modification, indicating that the lactose-modified electrode surface is significantly more hydrophilic. This is in agreement with previous results obtained following functionalization of sputtered amorphous carbon substrates [14] and may be attributed to the introduction of saccharide $-\mathrm{OH}$ moieties at the electrode interface. An even lower WCA of $27 \pm 3^{\circ}$ was observed for GC-Lac-E thus suggesting that electrografting yields a higher coverage of $-\mathrm{OH}$ groups than spontaneous grafting, in agreement with previous studies using aryldiazonium cations with small organic functionalities [32-35]. Multisolvent analysis was carried out to examine trends in surface free energy (SFE) using the Owen-Went-Rabel-Kaelble (OWRK) model to separate polar and dispersive contributions [41]. Three polar (water, glycerol and formamide) and two non-polar liquids (diiodomethane and 1- 
bromonaphthalene) with known reference surface tension components [42] were used for the analysis (Supporting Information). Table 1 summarizes the results which confirm that grafting of lactosides has a large effect on the polar component of the surface free energy (SFE): the polar contribution increases in the order GC $<$ GC-Lac-S $<$ GC-Lac-E, in agreement with increased wetting.
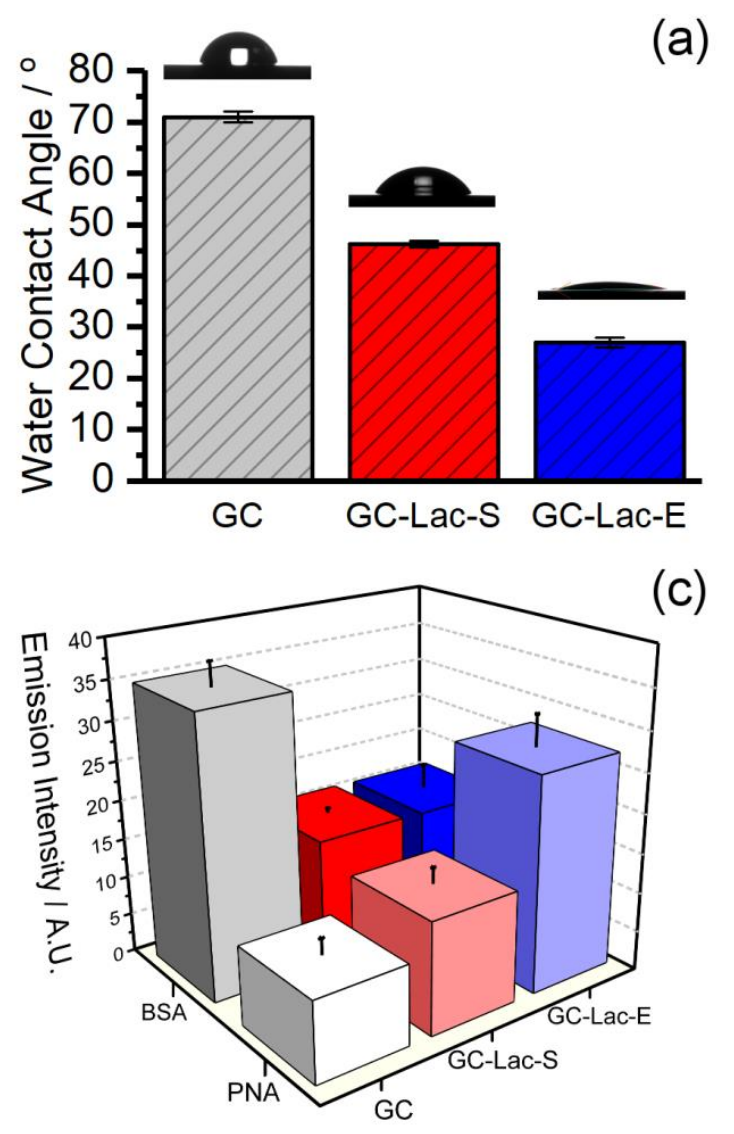

(c)

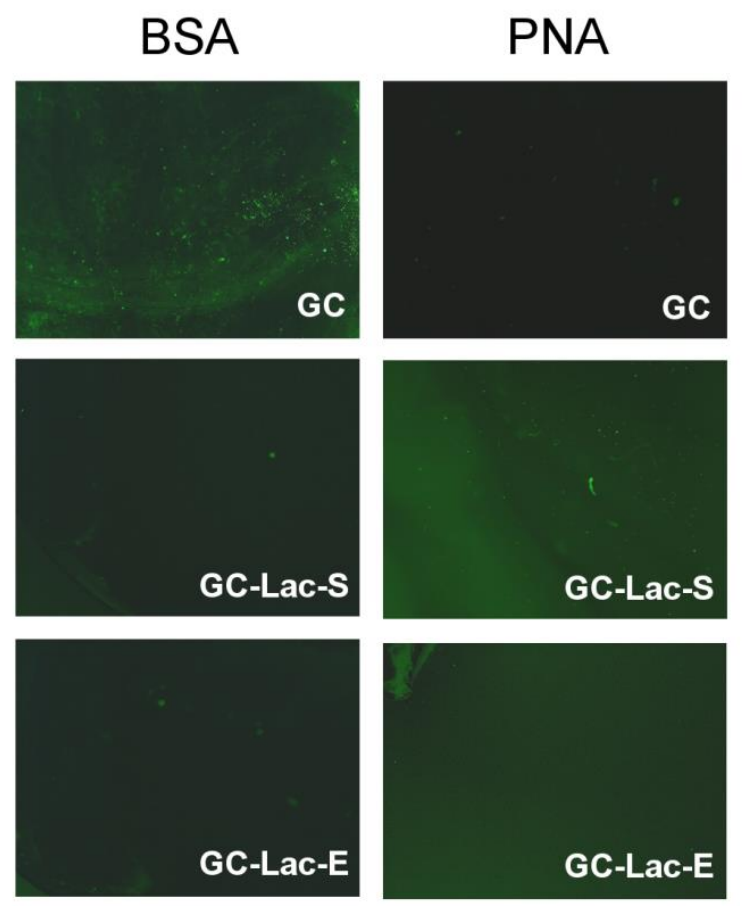

Figure 2. (a) Water contact angle for GC, GC-Lac-S and GC-Lac-E surfaces. (b) Fluorescence images of GC, GC-Lac-S and GC-Lac-E obtained after incubations in FITC conjugates of BSA and PNA; images are $2.7 \times 2.0 \mathrm{~mm}^{2}$. (c) Summary of average emission intensities obtained from the images in (b). 
Table 1. Surface free energy values for GC, GC-Lac-S and GC-Lac-E surfaces obtained using the OWRK model. The total free energy $\gamma^{\text {TOT }}$ is reported along with dispersive $\left(\gamma^{\mathrm{D}}\right)$ and polar components $\left(\gamma^{\mathrm{P}}\right)$.

\begin{tabular}{|l|c|c|c|}
\hline \multirow{2}{*}{ Surface } & \multicolumn{3}{|c|}{ SFE Components $\left(\mathbf{m J ~ m}^{-2}\right)$} \\
\cline { 2 - 4 } & $\gamma^{\mathbf{D}}$ & $\gamma^{\mathbf{P}}$ & $\gamma^{\text {TOT }}$ \\
\hline GC & $42.9 \pm 0.3$ & $4.9 \pm 0.5$ & $47.7 \pm 0.6$ \\
\hline GC-Lac-S & $43.8 \pm 0.2$ & $10.4 \pm 0.4$ & $54.2 \pm 0.4$ \\
\hline GC-Lac-E & $43.4 \pm 0.3$ & $19.8 \pm 0.8$ & $63.2 \pm 0.8$ \\
\hline
\end{tabular}

Fluorescence microscopy studies further support conclusions drawn from contact angle analysis; Figure 2b shows representative fluorescence images after immersion in FITC-BSA and FITC-PNA solutions of the three surfaces examined: GC, GC-Lac-S and GC-Lac-E. Figure 2c shows a summary of average fluorescence emission obtained at the three surfaces. The GC-LacE sample was found to display the highest overall emission intensity after the PNA-FITC treatment, whilst a lower intensity was observed for GC-Lac-S and almost no emission was observed on the unmodified GC surface. Given that PNA is a lectin with specific affinity for galactose units $[17,18,43]$, which are present at the solid-liquid interface after lactoside functionalization, this result suggests that a greater density of glycans is present on GC following electrografting compared to the spontaneous reaction. To exclude the possibility that this emission trend may be attributed to non-specific protein adhesion, we also carried out incubation experiments with solutions of BSA-FITC, which displays no specific affinity for lactosides but is a commonly-used protein foulant in adsorption studies [14, 17, 18]. Results in Figure $\mathbf{2 b}$ and 2c indicate that GC-Lac-S and GC-Lac-E display similar emission after immersion in BSA-FITC solutions, while the bare GC surface displays significant intensity, which is diagnostic of unspecific protein adsorption in the absence of any functional layer. Experiments with PNA and 
BSA therefore indicate that electrografting yields adlayers with significantly greater surface density of Lac units, as expected from multilayer formation via electrochemically assisted functionalization. However, despite significant differences in coverage, the reduction in BSA unspecific binding relative to the bare carbon electrode surface observed at the two Lacterminated surfaces is indistinguishable under the experimental conditions of the assay. Independently of whether Lac units are grafted spontaneously or electrochemically, GC-Lac surfaces appear equivalent in their ability to resist unspecific protein adsorption relative to bare GC electrodes.

To quantitatively compare the surface coverage of Lac units obtained via the two methodologies, XPS experiments were carried out on GC electrodes after functionalization in a solution containing a fluorine-substituted analogue of the aryl-lactoside precursor, shown in Figure 1. Fluorine substitution provides a convenient chemical marker for tracking the presence of covalently-grafted aryl-lactosides on substrates [18]. Figure $\mathbf{3}$ shows survey (3a), F 1s (3b) and C 1s (3c-e) spectra of GC, GC-Lac-S and GC-Lac-E; results obtained from best fits are summarized in Table 2. Survey scans of GC (Figure 3a) show only C 1s and O 1s peaks. Both GC-Lac-S and GC-Lac-E show an additional peak attributed to F 1s contributions. Figure 3b shows that the $\mathrm{F} 1 \mathrm{~s}$ peak position is at $c a .687 \mathrm{eV}$, characteristic of fluorinated organics in a low $\mathrm{F} / \mathrm{C} \%$ environment [44]. The F 1s intensity is significantly larger for GC-Lac-E and a comparison of the $\mathrm{F} / \mathrm{C}$ at.\% after normalization by sensitivity factors yields values of $0.4 \pm 0.2 \%$ for GC-Lac-S and $2.3 \pm 0.6 \%$ for GC-Lac-E (Table 2). This result confirms that electrografting produces an aryl-lactoside adlayer with a higher molecular surface density than the spontaneous one. 

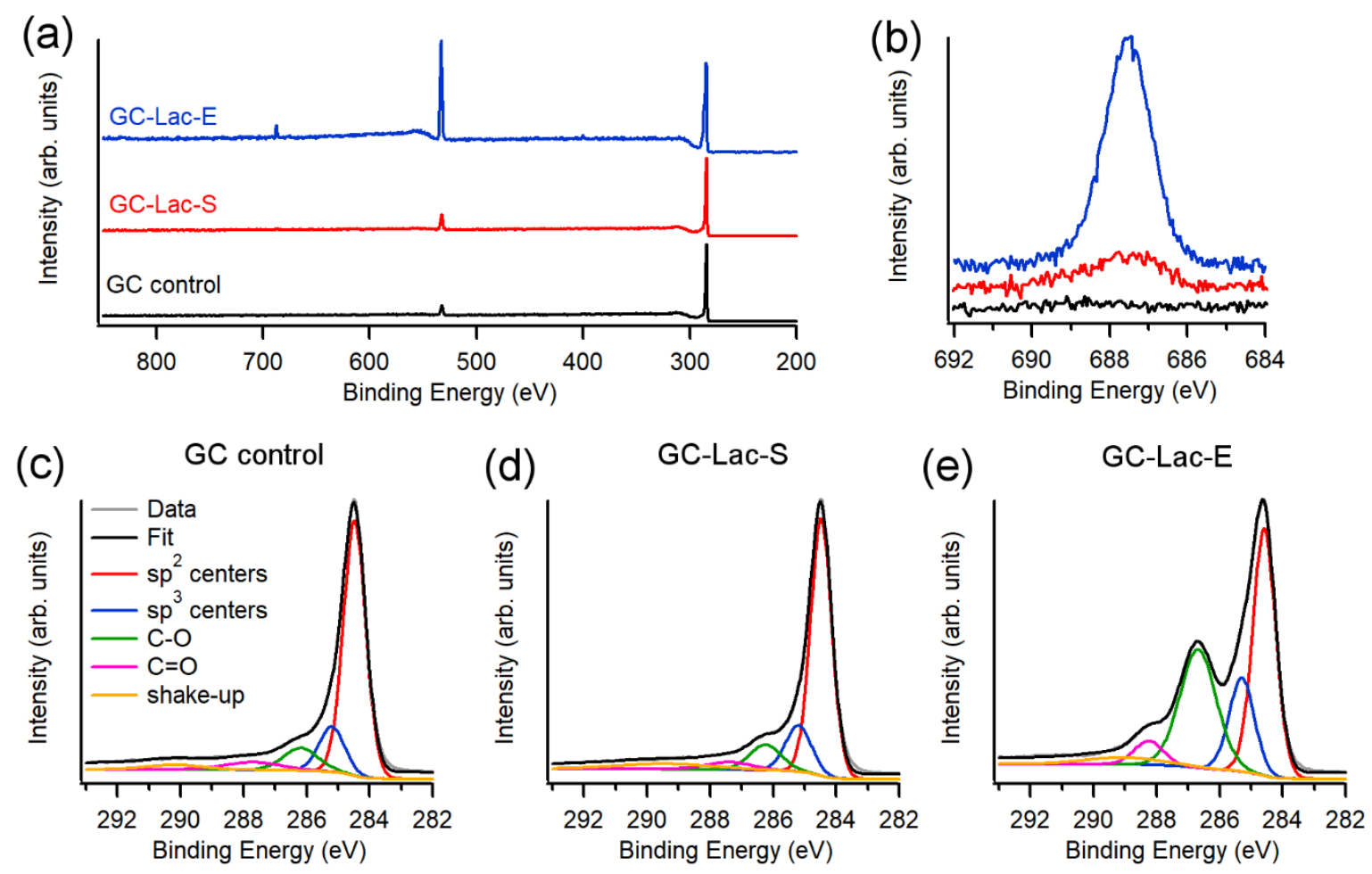

Figure 3. (a) XPS survey spectra of a control GC surface and of GC coated with a fluorinated derivative of the aryl-lactoside via spontaneous (GC F-Lac-S) and electrochemically assisted (GC-Lac-E) grafting. (b) F 1s region showing details of the fluorine contribution for the three surfaces. C 1s spectra of the control GC (c), GC-Lac-S (d) and GC-Lac-E (e) samples together with their deconvolution; in each case the raw data and the fitted envelope are offset for clarity.

Table 2. XPS parameters for GC, GC-Lac-S and GC-Lac-E surfaces derived from RSF-normalised area ratios of high resolution scans of the $\mathrm{F} 1 \mathrm{~s}, \mathrm{O} 1 \mathrm{~s}$ and $\mathrm{C} 1 \mathrm{~s}$ regions.

\begin{tabular}{|l|c|c|c|}
\hline Surface & $\mathbf{F} / \mathbf{C} \%$ & $\mathbf{O} / \mathbf{C} \%$ & $\mathbf{F} / \mathbf{C}-\mathbf{O} \%$ \\
\hline GC & - & $3 \pm 2$ & - \\
\hline GC-Lac-S & $0.4 \pm 0.2$ & $9 \pm 2$ & $2 \pm 1$ \\
\hline GC-Lac-E & $2.3 \pm 0.6$ & $27.9 \pm 0.6$ & $6 \pm 2$ \\
\hline
\end{tabular}

Differences in the aryl-lactoside coverage are also evident in the C 1s peaks, shown in Figure 3c (GC), 3d (GC-Lac-S) and 3e (GC-Lac-E) together with their deconvolution. All three surfaces 
have a predominant contribution at $c a .284 .5 \mathrm{eV}$ attributed to the graphitic carbon of the GC substrate; contributions from carbon atoms in the aryl ring of an aryl-lactoside in GC-Lac surfaces also fall in this region and cannot be resolved from those of the substrate. Higher binding energy contributions associated with tetrahedrally bonded carbon $(c a .285 .6 \mathrm{eV})$ and CO moieties $(c a .286 .5-288 \mathrm{eV})$ are visible for a bare GC. The C 1s envelope of GC-Lac-S surfaces remains similar to that of GC, suggesting that the $\mathrm{C} 1 \mathrm{~s}$ peak is dominated by photoemission from the carbon substrate as expected following formation of a thin adlayer. In the case of GC-Lac-E, contributions at. $286.5 \mathrm{eV}$ arising from the lactoside groups become very prominent. This change is accompanied by an increase in the $\mathrm{O} / \mathrm{C}$ content and a significant decrease in the fwhm of the O 1s peak, as shown in Supporting Information, thus indicating that oxidized groups present at the surface are chemically homogeneous.

Area normalization of the $\mathrm{F}$ 1s peak relative to the $286.5 \mathrm{eV}$ contribution from $\mathrm{C}-\mathrm{O}$ groups $\left(\mathrm{A}_{687} / \mathrm{A}_{286.6}\right)$ corrected by RSF values indicates that the atomic $\mathrm{F} / \mathrm{C}-\mathrm{O}=(6 \pm 2)$ at.\% (Table 2) for GC-Lac-E, i.e. close that expected from the molecular structure in Figure 1 of $8 \%$. By contrast GC-Lac-S samples yield F/C-O $=(2 \pm 1)$ at.\% which supports the presence of a thin adlayer on the GC surface after spontaneous grafting. The thickness of the electrografted aryllactoside layer was estimated from the $\mathrm{F} / \mathrm{C}$ at.\% value by calculating the expected attenuation of the carbon atom signal and considering the contributions from both the organic layer and the carbon substrate, as detailed in the Supporting Information. A thickness of $0.5 \mathrm{~nm}$ was estimated for the spontaneously grafted layer, in good agreement with previously reported thickness values obtained via AFM [14], whereas a thickness of $2.4 \mathrm{~nm}$ was obtained for the electrografted layer. This latter estimate was further supported by AFM experiments (see 
Supporting Information) that yielded an estimated thickness of $1.9 \pm 0.6 \mathrm{~nm}(95 \%$ confidence level) for electrografted layers.

\subsection{Charge transfer at GC-Lac Electrodes}

Spectroscopic studies of GC-Lac electrodes suggest that the amount of lactoside on each electrode surface differs significantly and is likely due to multilayer versus monolayer coverage on GC-Lac-E and GC-Lac-S, respectively. To investigate the effects of these two types of glycan layers on the charge transfer properties of the GC electrodes, we carried out cyclic voltammetry studies in solutions containing standard redox probes. Two redox couples were chosen: hexaammine ruthenium $\mathrm{Ru}\left(\mathrm{NH}_{3}\right)_{6}^{2+/ 3+}$ and potassium hexacyanoferrate $\mathrm{Fe}(\mathrm{CN}) 6^{3-/ 4-}$. As described in a previous publication [37], the $\mathrm{Ru}\left(\mathrm{NH}_{3}\right)_{6}{ }^{2+/ 3+}$ redox couple is an outer-sphere couple, i.e. its heterogeneous charge transfer properties are insensitive to the chemical termination of the electrode surface and only sensitive to bulk electronic properties and coupling of acceptor-donor states, whilst $\mathrm{Fe}(\mathrm{CN}) 6^{3-4-}$ is sensitive to surface termination [45].

Figure 4a shows representative cyclic voltammograms (CVs) of both GC-Lac electrodes and unmodified $\mathrm{GC}$ in $1.0 \mathrm{mM}$ solutions of $\mathrm{Ru}\left(\mathrm{NH}_{3}\right)_{6}{ }^{3+}$ in $0.1 \mathrm{M} \mathrm{KCl}$ at $10 \mathrm{mV} \mathrm{s}^{-1}$; peak-to-peak separation $\left(\Delta \mathrm{E}_{\mathrm{p}}\right)$ values determined for each electrode are summarized in Table 3. It is evident from the overlap of the voltammograms in the figure that all three electrodes have similar charge transfer properties to $\mathrm{Ru}\left(\mathrm{NH}_{3}\right)_{6}{ }^{3+}$. Furthermore, all three electrodes show $\Delta \mathrm{E}_{\mathrm{p}}$ values close to the theoretical value of $59 \mathrm{mV}$ for a reversible $1 \mathrm{e}$ transfer at $25{ }^{\circ} \mathrm{C}$; the $\Delta \mathrm{E}_{\mathrm{p}}$ remains close to the theoretical value even at faster scan rates (see Supporting Information), indicating that the charge transfer kinetics are rapid on GC and remain unaffected by the presence of either spontaneous or electrografted lactoside coatings. 

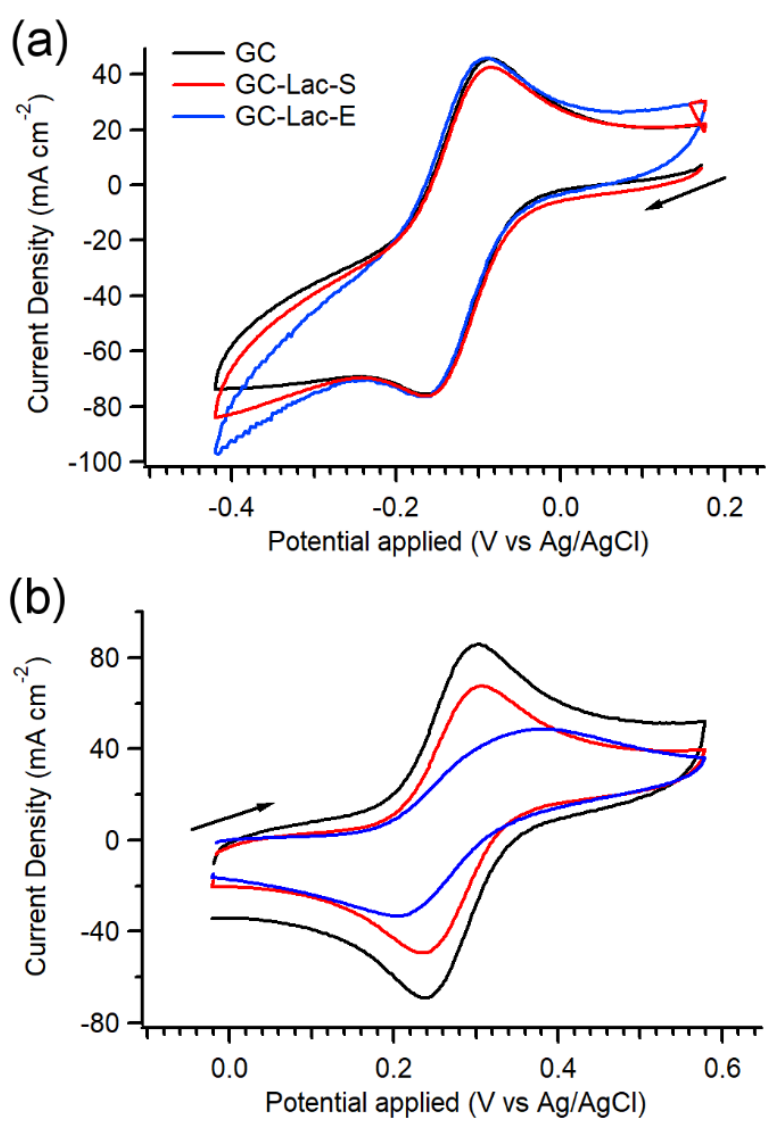

Figure 4. Cyclic voltammograms of GC and GC-Lac-S electrodes in solutions of (a) $1.0 \mathrm{mM}$ $\mathrm{Ru}\left(\mathrm{NH}_{3}\right)_{6}{ }^{3+} / 0.1 \mathrm{M} \mathrm{KCl}$ and (b) $1.0 \mathrm{mM} \mathrm{Fe}\left(\mathrm{CN}_{6}\right)^{4-} / 0.1 \mathrm{M} \mathrm{KCl}$. Scan rate: $10 \mathrm{mV} \mathrm{s}^{-1}$.

Table 3. Summary of $\Delta \mathrm{E}_{\mathrm{p}}$ values (in $\mathrm{mV}$ ) for GC and GC-Lac surfaces in $\mathrm{Ru}\left(\mathrm{NH}_{3}\right)_{6}{ }^{3+}$ and $\mathrm{Fe}\left(\mathrm{CN}_{6}\right)^{4-}$ solutions in $0.1 \mathrm{M} \mathrm{KCl}$ obtained at $10 \mathrm{mV} \mathrm{s}^{-1}$. Errors represent $95 \%$ confidence intervals.

\begin{tabular}{|l|c|c|}
\hline \multicolumn{1}{|c|}{ Surface } & $\begin{array}{c}\Delta \mathbf{E}_{\mathbf{p}}(\mathbf{m V}) \\
\text { in } \mathbf{R u}\left(\mathbf{N H}_{\mathbf{3}}\right)^{{ }^{3+}}\end{array}$ & $\begin{array}{c}\Delta \mathbf{E}_{\mathbf{p}}(\mathbf{m V}) \\
\text { in } \mathbf{F e}\left(\mathbf{C N}_{\mathbf{6}}\right)^{{ }^{4-}}\end{array}$ \\
\hline GC & $63 \pm 6$ & $64 \pm 6$ \\
\hline GC-Lac-S & $64 \pm 10$ & $66 \pm 6$ \\
\hline GC-Lac-E & $61 \pm 14$ & $140 \pm 30$ \\
\hline
\end{tabular}

The cyclic voltammograms at GC-Lac-S and GC-Lac-E differ however significantly in 1.0 $\mathrm{mM} \mathrm{Fe}(\mathrm{CN})^{3-/ 4-}$ solution, as shown in Figure $4 \mathbf{b}$. The $\Delta \mathrm{E}_{\mathrm{p}}$ of $66 \pm 6 \mathrm{mV}$ observed on the GC- 
Lac-S surface is close to the reversible limit as discussed for $\mathrm{Ru}\left(\mathrm{NH}_{3}\right)_{6}{ }^{3+}$ above and indistinguishable from the value of $64 \pm 6 \mathrm{mV}$ found on unmodified GC (Table 3); CVs obtained at faster scan rate show that the change in $\Delta \mathrm{E}_{\mathrm{p}}$ remains modest even at faster scan rates (see Supporting Information). The current densities observed on GC and GC-Lac-S are also comparable. These data suggest that spontaneous grafting of aryl-lactosides does not significantly impede charge transfer to the surface-sensitive $\mathrm{Fe}(\mathrm{CN})_{6}^{3-/ 4-}$ redox probe. On the contrary, the CV of GC-Lac-E electrodes shows a large apparent $\Delta \mathrm{E}_{\mathrm{p}}$ of $140 \pm 30 \mathrm{mV}$ and a significant depression in the peak current density relative to both GC and GC-Lac-S electrodes. Such behavior strongly suggests that charge transfer to $\mathrm{Fe}(\mathrm{CN}) 6^{3 / 4-}$ is impeded by the presence of the lactoside layer and is only able to occur at specific regions of the GC-Lac-E surface, likely defects/pinholes in the saccharide layer, as observed for instance for $\mathrm{Fe}(\mathrm{CN}) 6^{3-/ 4-}$ on $\mathrm{Au}$ electrodes functionalized with self-assembled monolayers [46]. Previous work on the voltammetry of $\mathrm{Fe}(\mathrm{CN})_{6}^{3-/ 4-}$ at electrodes modified via aryldiazonium grafting has shown that electrostatic repulsion/attraction can also contribute to the observed response when moieties amenable to protonation are introduced via functionalisation [38]. However aryl-lactosides do not possess ionizable groups at neutral $\mathrm{pH}$ and their effects on surface charge densities have been experimentally shown to be negligible in previous work [14]. Therefore, it is likely that the passivation observed is primarily due to the barrier effect of the electrografted film. In summary, the grafting protocol has a profound effect on charge transfer through the aryl-lactoside layer, with spontaneously deposited layers being "invisible" to the electrochemical response, whereas electrografted layers significantly inhibit transfer kinetics to moderately surface-sensitive redox species. 


\subsection{Performance of GC-Lac electrodes in complex matrices}

The above findings, in combination with protein resistance results, suggest that it is possible to preserve fast redox kinetics at spontaneously modified GC-Lac-S electrodes, while displaying similar protein resistance when compared to electrografted GC-Lac-E surfaces. However, studies using BSA solutions in buffer are far from conditions found in real samples of interest in electroanalytical applications. Electrochemical probes and biosensors are often exposed to numerous foulants with a range of concentrations over an extended period of use. To test whether the results obtained with standard redox probes and single protein solutions can be translated to applications in complex matrices, we tested the detection of a redox species in a realistic food sample after prolonged exposure of the electrode surface to the matrix.

Recently, Redivo et al. showed that bare glassy carbon electrodes can be effective probes for the electrochemical determination of caffeine concentration in commercial beverages including coffee [47]. Coffee presents a significant challenge in electroanalysis as it is comprised of thousands of components spanning a range of molecular weights, which can result in fouling of probe surfaces [48]. Figure 5a (solid line) shows a representative CV for our bare GC electrode in a standard solution of $1.0 \mathrm{mM}$ caffeine in $0.1 \mathrm{M} \mathrm{H}_{2} \mathrm{SO}_{4}$; the caffeine oxidation peak appears at $c a .1 .50 \mathrm{~V}$ vs $\mathrm{Ag} / \mathrm{AgCl}$. Similar results were obtained for both GC-Lac-S and GC-Lac-E electrodes (Figure 5b and 5c), suggesting that the presence of the lactose coating does not significantly interfere with caffeine oxidation. The electrodes were immersed over a $72 \mathrm{~h}$ period in a coffee solution freshly prepared as indicated in the experimental section. Following incubation, the electrodes were rinsed gently for ten seconds with deionised water under gravity [18] and the voltammetry experiment was repeated under identical conditions. The post-immersion voltammograms are reported as dashed lines in Figures 5a-c. Both unmodified 
GC and the GC-Lac electrodes show increased capacitive current after the immersion, but the effect is most pronounced on the bare GC, leading to a masking of the oxidative caffeine peak and hence lower sensitivity for electrochemical caffeine detection. Both GC-Lac-S and GC-LacE samples show only a minor change after the immersion, suggesting that both of these probes are resistant to non-specific biofouling from prolonged exposure to a complex biological matrix.
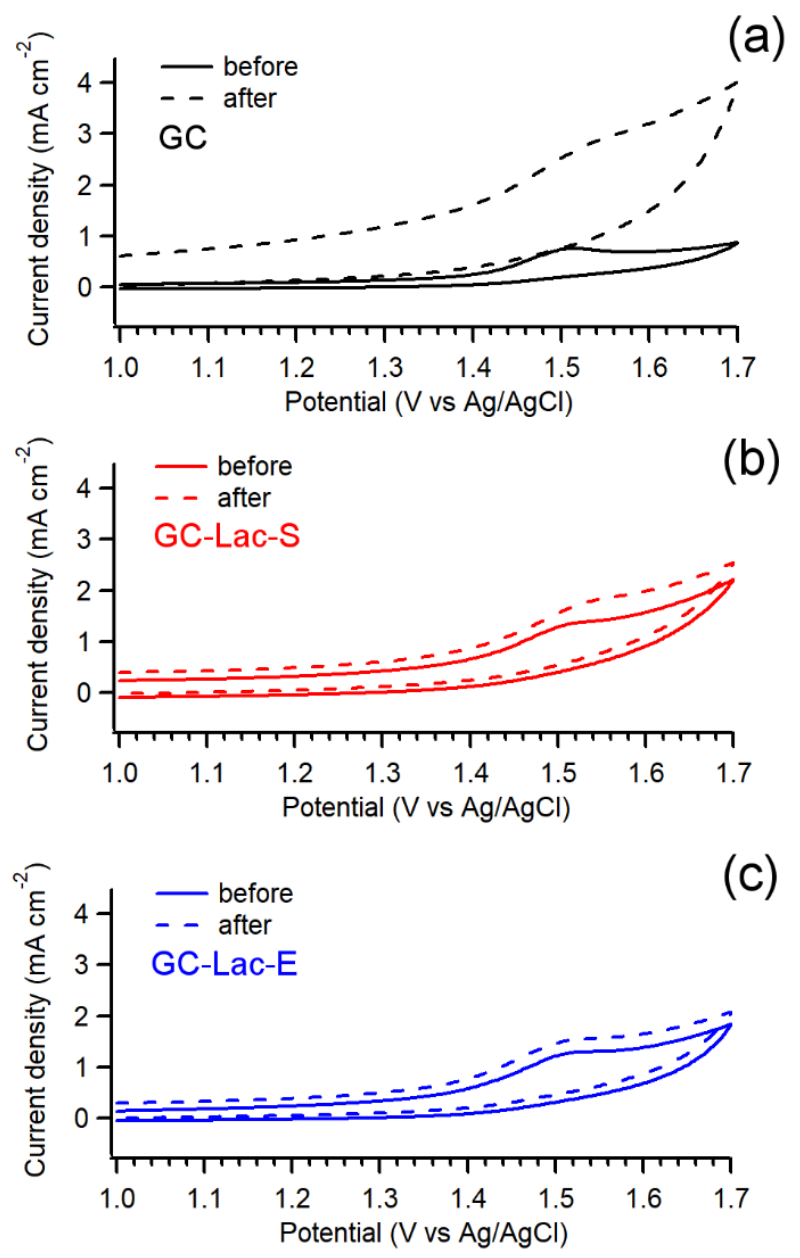

Figure 5. Cyclic voltammograms of $1.0 \mathrm{mM}$ caffeine in $0.1 \mathrm{M} \mathrm{H}_{2} \mathrm{SO}_{4}$ obtained at $50 \mathrm{mV} \mathrm{s}^{-1}$ using (a) GC, (b) GC-Lac-S and (c) GC-Lac-E electrodes before (solid lines) and after (dashed lines) immersion in a commercial coffee solution for $72 \mathrm{~h}$. The second CV scan is reported in each case. 


\section{Conclusions}

We have functionalized GC electrodes with aryl-lactoside coatings using aryldiazonium chemistry via both spontaneous grafting and electrografting methods. Fluorescence and XPS studies suggest that spontaneous grafting produces a monolayer or sub-monolayer aryl-lactoside coating on the surface whilst electrografting produces a multilayer. Both coatings are anti-fouling towards solutions of BSA compared to unmodified GC which fouls significantly under the same conditions. Spontaneous grafting was shown to not interfere with charge transfer to the surface-sensitive $\mathrm{Fe}(\mathrm{CN}) 6^{3-/ 4-}$ redox probe in cyclic voltammetry studies, whilst the electrografted multilayer inhibits charge transfer displaying a passivating effect.

All three electrodes were tested as electrochemical sensors for the detection of caffeine, but only the lactoside coated electrodes are capable of resisting biofouling after extended exposure to commercial coffee samples. Surface free energy determination for each surface indicates that both lactoside coatings present surfaces with large polar contributions, attributed to enhanced hydrophilicity of the saccharide layer. Increased hydrophilicity is likely at the origin of the resistance to fouling displayed by both surfaces. Spontaneous diazonium grafting of aryl lactosides is therefore a convenient method for the preparation of biomimetic carbon electrodes with good antifouling performance in complex matrices whilst preserving electro-permeability. We expect these electrodes to perform well in the detection of other electrochemical probe/biosensor applications in biological media; such studies with different redox analytes are currently underway in our laboratory. 


\section{Acknowledgements}

This publication has emanated from research conducted with the financial support of Science Foundation Ireland (SFI) grant No. 13/CDA/2213. AM and JAB gratefully acknowledge support from the School of Chemistry and the Irish Research Council Grant No. GOIPG/2014/399, respectively. EW is grateful for support by the Undergraduate Research Bursary Program of the Royal Society of Chemistry and Nuffield Foundation. This project has received funding from the European Union's Horizon 2020 research and innovation programme under the Marie Skłodowska-Curie grant agreement No. 799175 (HiBriCarbon). The results of this publication reflect only the authors' view and the Commission is not responsible for any use that may be made of the information it contains.

Supporting Information. CV and chronoamperograms obtained in diazotization solutions; multisolvent contact angle data and details of the model used to calculate polar and dispersive components of the solid surface tensions.

\section{References}

[1] Varki A, Lowe JB. Biological roles of glycans. In: Cummings RD, Esko JD, Stanley P, Bertozzi C, Hart GW, Etzler ME, eds. Essentials of Glycobiology 2nd edition. New York: Cold Spring Harbor Laboratory Press 2009.

[2] Varki A. Biological roles of glycans. Glycobiology. 2016;27(1):3-49.

[3] Kang B, Opatz T, Landfester K, Wurm FR. Carbohydrate nanocarriers in biomedical applications: functionalization and construction. Chem Soc Rev. 2015;44(22):8301-25.

[4] Gorityala BK, Ma J, Wang X, Chen P, Liu X-W. Carbohydrate functionalized carbon nanotubes and their applications. Chem Soc Rev. 2010;39(8):2925-34. 
[5] Marradi M, Chiodo F, García I, Penadés S. Glyconanoparticles as multifunctional and multimodal carbohydrate systems. Chem Soc Rev. 2013;42(11):4728-45.

[6] García I, Mosquera J, Plou J, Liz-Marzán LM. Plasmonic Detection of CarbohydrateMediated Biological Events. Adv Opt Mater. 2018;6(23):1800680.

[7] Delbianco M, Bharate P, Varela-Aramburu S, Seeberger PH. Carbohydrates in Supramolecular Chemistry. Chem Rev. 2016;116(4):1693-752.

[8] Barrière F, Downard AJ. Covalent modification of graphitic carbon substrates by nonelectrochemical methods. J Solid State Electrochem. 2008;12(10):1231-44.

[9] Adenier A, Barré N, Cabet-Deliry E, Chaussé A, Griveau S, Mercier F, et al. Study of the spontaneous formation of organic layers on carbon and metal surfaces from diazonium salts. Surf Sci. 2006;600(21):4801-12.

[10] Adenier A, Cabet-Deliry E, Chaussé A, Griveau S, Mercier F, Pinson J, et al. Grafting of Nitrophenyl Groups on Carbon and Metallic Surfaces without Electrochemical Induction. Chem Mater. 2005;17(3):491-501.

[11] Hurley BL, McCreery RL. Covalent Bonding of Organic Molecules to $\mathrm{Cu}$ and Al Alloy 2024 T3 Surfaces via Diazonium Ion Reduction. J Electrochem Soc. 2004;151(5):B252-B9.

[12] Cullen RJ, Jayasundara DR, Soldi L, Cheng JJ, Dufaure G, Colavita PE. Spontaneous Grafting of Nitrophenyl Groups on Amorphous Carbon Thin Films: A Structure-Reactivity Investigation. Chem Mater. 2012;24(6):1031-40.

[13] Jayasundara DR, Duff T, Angione MD, Bourke J, Murphy DM, Scanlan EM, et al. Carbohydrate Coatings via Aryldiazonium Chemistry for Surface Biomimicry. Chem Mater. 2013;25(20):4122-8.

[14] Zen F, Angione MD, Behan JA, Cullen RJ, Duff T, Vasconcelos JM, et al. Modulation of Protein Fouling and Interfacial Properties at Carbon Surfaces via Immobilization of Glycans Using Aryldiazonium Chemistry. Sci Rep. 2016;6:24840.

[15] Zen F, Karanikolas VD, Behan JA, Andersson J, Ciapetti G, Bradley AL, et al. Nanoplasmonic Sensing at the Carbon-Bio Interface: Study of Protein Adsorption at Graphitic and Hydrogenated Carbon Surfaces. Langmuir. 2017;33(17):4198-206.

[16] Angione MD, Duff T, Bell AP, Stamatin SN, Fay C, Diamond D, et al. Enhanced Antifouling Properties of Carbohydrate Coated Poly(ether sulfone) Membranes. ACS Appl Mater Interfaces. 2015;7(31):17238-46.

[17] Esteban-Tejeda L, Duff T, Ciapetti G, Daniela Angione M, Myles A, Vasconcelos JM, et al. Stable hydrophilic poly(dimethylsiloxane) via glycan surface functionalization. Polymer. 2016;106:1-7. 
[18] Myles A, Haberlin D, Esteban-Tejeda L, Angione MD, Browne MP, Hoque MK, et al. Bioinspired Aryldiazonium Carbohydrate Coatings: Reduced Adhesion of Foulants at Polymer and Stainless Steel Surfaces in a Marine Environment. ACS Sustain Chem Eng. 2018;6(1):114151 .

[19] Myles A, Behan JA, Twamley B, Colavita PE, Scanlan EM. Spontaneous Aryldiazonium Grafting for the Preparation of Functional Cyclodextrin-Modified Materials. ACS Applied Bio Materials. 2018;1(3):825-32.

[20] Kissinger P, Heineman WR. Laboratory Techniques in Electroanalytical Chemistry, revised and expanded: CRC press; 1996.

[21] McCreery RL. Advanced carbon electrode materials for molecular electrochemistry. Chem Rev. 2008;108(7):2646-87.

[22] Balasubramanian K, Burghard M. Biosensors based on carbon nanotubes. Anal Bioanal Chem. 2006;385(3):452-68.

[23] Jaiswal N, Tiwari I. Recent build outs in electroanalytical biosensors based on carbonnanomaterial modified screen printed electrode platforms. Anal Methods. 2017;9(26):3895-907.

[24] Takmakov P, Zachek MK, Keithley RB, Walsh PL, Donley C, McCarty GS, et al. Carbon Microelectrodes with a Renewable Surface. Anal Chem. 2010;82(5):2020-8.

[25] Chang A-Y, Dutta G, Siddiqui S, Arumugam PU. Surface Fouling of Ultrananocrystalline Diamond Microelectrodes during Dopamine Detection: Improving Lifetime via Electrochemical Cycling. ACS Chem Neurosci. 2019;10(1):313-22.

[26] Jackowska K, Krysinski P. New trends in the electrochemical sensing of dopamine. Anal Bioanal Chem. 2013;405(11):3753-71.

[27] Yang C, Wang Y, Jacobs CB, Ivanov IN, Venton BJ. O2 Plasma Etching and Antistatic Gun Surface Modifications for CNT Yarn Microelectrode Improve Sensitivity and Antifouling Properties. Anal Chem. 2017;89(10):5605-11.

[28] Belghiti DK, Scorsone E, de Sanoit J, Bergonzo P. Simultaneous detection of indole and 3methylindole using boron-doped diamond electrodes. Phys Status Solidi A. 2016;213(10):266271.

[29] Bélanger D, Pinson J. Electrografting: a powerful method for surface modification. Chem Soc Rev. 2011;40(7):3995-4048.

[30] Brooksby PA, Downard AJ. Electrochemical and Atomic Force Microscopy Study of Carbon Surface Modification via Diazonium Reduction in Aqueous and Acetonitrile Solutions. Langmuir. 2004;20(12):5038-45. 
[31] Delamar M, Hitmi R, Pinson J, Saveant JM. Covalent modification of carbon surfaces by grafting of functionalized aryl radicals produced from electrochemical reduction of diazonium salts. J Am Chem Soc. 1992;114(14):5883-4.

[32] Kariuki JK, McDermott MT. Nucleation and Growth of Functionalized Aryl Films on Graphite Electrodes. Langmuir. 1999;15(19):6534-40.

[33] Kariuki JK, McDermott MT. Formation of Multilayers on Glassy Carbon Electrodes via the Reduction of Diazonium Salts. Langmuir. 2001;17(19):5947-51.

[34] Doppelt P, Hallais G, Pinson J, Podvorica F, Verneyre S. Surface Modification of Conducting Substrates. Existence of Azo Bonds in the Structure of Organic Layers Obtained from Diazonium Salts. Chem Mater. 2007;19(18):4570-5.

[35] Downard AJ. Electrochemically Assisted Covalent Modification of Carbon Electrodes. Electroanalysis. 2000;12(14):1085-96.

[36] Behan JA, Hoque MK, Stamatin SN, Perova TS, Vilella-Arribas L, García-Melchor M, et al. Experimental and Computational Study of Dopamine as an Electrochemical Probe of the Surface Nanostructure of Graphitized N-Doped Carbon. J Phys Chem C. 2018;122(36):2076373.

[37] Behan JA, Stamatin SN, Hoque MK, Ciapetti G, Zen F, Esteban-Tejeda L, et al. Combined Optoelectronic and Electrochemical Study of Nitrogenated Carbon Electrodes. J Phys Chem C. 2017;121(12):6596-604.

[38] Richard W, Evrard D, Gros P. Kinetic Study of Redox Probes on Glassy Carbon Electrode Functionalized by 4-nitrobenzene Diazonium. Int J Electrochem Sci. 2019;14:453-69.

[39] Behan JA, Grajkowski F, Jayasundara DR, Vilella-Arribas L, García-Melchor M, Colavita PE. Influence of carbon nanostructure and oxygen moieties on dopamine adsorption and charge transfer kinetics at glassy carbon surfaces. Electrochim Acta. 2019;304:221-30.

[40] Behan JA, Iannaci A, Domínguez C, Stamatin SN, Hoque MK, Vasconcelos JM, et al. Electrocatalysis of $\mathrm{N}$-doped carbons in the oxygen reduction reaction as a function of $\mathrm{pH}$ : $\mathrm{N}$-sites and scaffold effects. Carbon. 2019;148:224-30.

[41] Owens DK, Wendt RC. Estimation of the surface free energy of polymers. J Appl Polym Sci. 1969;13(8):1741-7.

[42] van Oss CJ. Interfacial Forces in Aqueous Media. 1st ed. New York: Taylor \& Francis; 1994.

[43] Maupin KA, Liden D, Haab BB. The fine specificity of mannose-binding and galactosebinding lectins revealed using outlier motif analysis of glycan array data. Glycobiology.

2012;22(1):160-9. 
[44] Dilks A, Kay E. Plasma polymerization of ethylene and the series of fluoroethylenes: plasma effluent mass spectrometry and ESCA studies. Macromolecules. 1981;14(3):855-62.

[45] Granger MC, Swain GM. The Influence of Surface Interactions on the Reversibility of Ferri/Ferrocyanide at Boron-Doped Diamond Thin-Film Electrodes. J Electrochem Soc. 1999;146(12):4551-8.

[46] Janek RP, Fawcett WR, Ulman A. Impedance Spectroscopy of Self-Assembled Monolayers on $\mathrm{Au}(111)$ : Sodium Ferrocyanide Charge Transfer at Modified Electrodes. Langmuir. 1998;14(11):3011-8.

[47] Redivo L, Stredanský M, De Angelis E, Navarini L, Resmini M, Švorc Ĺ. Bare carbon electrodes as simple and efficient sensors for the quantification of caffeine in commercial beverages. Roy Soc Open Sci. 2018;5(5).

[48] Clarke RJ. Coffee: Volume 1: Chemistry: Springer Netherlands; 2012. 